\title{
On some reasons why the time reversal operator could be unitary
}

\author{
N. Debergh ${ }^{\mathrm{a}, *}$, J.-P. Petit ${ }^{\mathrm{b}}$ \\ a Department of pedagogy. Haute Ecole Charlemagne, 1, rue Grégoire Bodart, 4500 Huy. Belgium \\ ${ }^{b}$ Centre National de la Recherche Scien tifique (ONRS). France ${ }^{1}$ \\ Received 4 October 2021 \\ 4ccepted 22 October 2021
}

\begin{abstract}
Keywords:
Time reversal operator

Discrete symmetries in non-relativistic

quantum mechanics
\end{abstract}

\begin{abstract}
A B S T R A C T
The time reversal operator can be unitary or anti-unitary. In the first case, there are necessarily states of negative energy, whereas they do not appear in the second case. For a long time it was believed that these negative energies were irrelevant, until the discovery of the acceleration of the expansion of the universe gave them a meaning. We examine the possibility for the time inversion to be associated with a unitary operator in the quantum level by looking at the usual arguments in favor of the anti-unitarity of this operator and by proposing counter-arguments in order to validate the possibility for this operator to be unitary.
\end{abstract}

\section{Introduction}

In his famous book The Quantum Theory of Fields, Steven Weinberg quoted that if the time reversal operator $T$ is supposed to be linear and unitary, then "for any state $\psi$ of energy $E$, there is another state $T^{-1} \psi$ of energy $-E^{\prime \prime}[1]$. At the time Weinberg wrote this sentence, there was no reason to believe that negative energy states made sense. Indeed, there was no phenomenon, be it experiments or cosmological observations, which allowed to think that these states could play a significant role or even exist. It was therefore decided by the physics community that $T$ would be an anti-unitary operator, so as not to make these negative energy states appear.

However, three years after the publication of Weinberg's book, two teams of astronomers, respectively led by Saul Perlmutter and Brian P. Schmidt, have reached the unexpected result that the expansion of the Universe seems to be accelerating [2]. The Nobel Prize was awarded to them and to Adam G. Riess, in 2011, for this discovery which implies the existence of an unknown form of matter -called dark energy- whose pressure would be negative, with a repulsive and not attractive behavior towards gravitation.

If the nature of this dark energy is, at present, one of the biggest issues submitted to the sagacity of researchers, there is more and more evidence to say that particles of negative masses ([3-7]) (equivalent to negative energies in the relativistic context) are one

\footnotetext{
* Corresponding author. E-mail address: nathalie.debergh@hech.be (N. Debergh).

1 Retired from.
}

of the best candidates to specify this nature ([8,9]). In fact, dark energy would be the sum of negative energy states associated to negative mass particles.

As these particles interact only by antigravity with positive mass matter and not through electromagnetic phenomena, it is not possible to demonstrate them from an experimental point of view. The detection is done indirectly, by analyzing the alteration of the light emitted by the particles of positive mass. We refer the interested reader to [10] where he can find the observational confirmations of this hypothesis for dark matter as well as dark energy, at the cosmological scale.

Returning to Weinberg's statement, it is therefore relevant, twentyseven years after it was written, to come back to the belief that states of negative energy cannot exist and to its theoretical counterpart i.e. the unitarity of $T$. If cosmological observations tend to prove that negative energy states have a physical meaning, we should now examine the relevance of a unitary time reversal operator at the quantum mechanical level. This is precisely the purpose of this article.

To do this, we briefly review the definitions related to unitary and anti-unitary operators in Section 2. We take a look at what happens with the time inversion in classical mechanics in Section 3. We then go to quantum mechanics in Section 4 and point out three arguments, frequently found in the standard literature, for why $T$ should be anti-unitary. To these three arguments, we oppose counter-arguments arguing for the unitarity of $T$. We also show that considering $T$ as a unitary operator has the same effect as applying the complex rotation method. This will be achieved in Section 5 as well as the introduction of a new inner product. We 
finally conclude by listing in a table the characteristics of the two options: $T$ unitary or anti-unitary.

\section{Definitions}

Since Wigner's symmetry representation theorem [11], we know that a symmetry operator i.e. an operator preserving transition probabilities, is necessarily unitary or anti-unitary.

As a reminder, a unitary operator $T$ is such that

$T^{\dagger} T=T T^{\dagger}=I$

( $T^{\dagger}$ being the adjoint of $T$ ).

Linearity is defined through

$T(c 1|\psi 1\rangle+c 2|\psi 2\rangle)=c 1 T|\psi 1\rangle+c 2 T|\psi 2\rangle$,

$c 1, c 2 \in \mathbb{C}, \forall|\psi 1\rangle,|\psi 2\rangle$.

Such an operator preserves the scalar product (and thus the probabilities)

$\langle\psi 1 \mid \psi 2\rangle=\langle T \psi 1 \mid T \psi 2\rangle$

An anti-unitary operator returns the complex conjugate of this scalar product (but therefore also preserves the probabilities)

$\langle\psi 1 \mid \psi 2\rangle^{*}=\langle T \psi 1 \mid T \psi 2\rangle$

It is thus anti-linear

$T(c 1|\psi 1\rangle+c 2|\psi 2\rangle)=c 1^{*} T|\psi 1\rangle+c 2^{*} T|\psi 2\rangle, \forall|\psi 1\rangle,|\psi 2\rangle$

This theorem has since been generalized under the name of Uhlhorn's theorem [12]. The latter is based on a less restrictive assumption: it is sufficient to request that

$\langle\psi 1 \mid \psi 2\rangle=0 \Longleftrightarrow\langle T \psi 1 \mid T \psi 2\rangle=0$

to ensure that $T$ is either unitary or anti-unitary.

In any case, the conclusion is the same: a symmetry operator is necessarily unitary or anti-unitary.

When the symmetry concerns continuous transformations (translations, rotations, ...), the choice is obvious: the operator is necessarily unitary. The reason is simple. For these transformations, we have:

$U(a) U(b)=U(c)$

For instance, let us consider the transformation

$U(a):|\psi(x)\rangle \rightarrow|\psi(x-a)\rangle$

representing the translation, according to the parameter $a$, in the wave functions space. If we expand $|\psi(x-a)\rangle$ with a Taylor series expansion, we get

$|\psi(x)\rangle-a \frac{d}{d x}|\psi(x)\rangle+\ldots+(-1)^{n} a^{n} \frac{d^{n}}{d x^{n}}|\psi(x)\rangle+\ldots=e^{-a \frac{d}{d x}}|\psi(x)\rangle$

Thus, in this case, we identify

$U(a) \equiv e^{-a \frac{d}{d x}}$

It is then obvious that $U(a) U(b)=U(c)$ is true with $c=a+b$.

Now, due to this result, a composition of two unitary operators as well as a composition of two anti-unitary operators is automatically unitary. This fixes univocally the unitarity of $U$.

The same is not true for discrete symmetries, of which time reversal is a part. In this case, the nature of the symmetry operator is left to the choice of the physicists and, as far as time reversal is concerned, this choice is (almost) unanimously fixed as being the anti-unitarity.

From now on, we investigate the other possibility: $T$ is unitary.

\section{A little detour through classical mechanics}

The time inversion in classical mechanics is defined by

$t \rightarrow-t$

and its impact on the variables of the phase space is

$\vec{r} \rightarrow \vec{r}, \vec{v}=\frac{d \vec{r}}{d t} \rightarrow-\vec{v}$

It is customary to consider indifferently the velocity $\vec{v}$ or the momentum $\vec{p}$ to characterize the phase space. Yet, in the case at hand i.e. the consideration of time reversal, the difference is going to be crucial. Indeed, there are as many expressions of momentum in classical mechanics as there are Lagrangians or Hamiltonians, whereas there is only one for velocity.

For example the Lagrangian of classical electrodynamics is given by

$L=T-V=\frac{1}{2} m \vec{v} \cdot \vec{v}-q \phi+q \vec{v} \cdot \vec{A}$

( $q$ is the electric charge, $\phi$ the scalar potential, and $\vec{A}$ the vector potential). Momenta are defined by

$\vec{p}=\frac{\partial L}{\partial \vec{v}}$

which gives rise here to

$\vec{p}=m \vec{v}+q \vec{A}$

The only way to ensure that the momentum changes sign under time reversal is thus to force the potential vector to change sign, too. However, if we consider f.i. the (frequently used) case of a constant magnetic field

$\vec{B}=B \vec{e}_{z}, B=$ constant

and the associated vector potential

$\vec{A}=-\frac{B}{2} \vec{e}_{x}+\frac{B}{2} \vec{e}_{y}$

we see clearly that this constraint of change of sign does not make sense.

Consequently, and in agreement with Albert [13], we conclude that the momentum does not necessarily change sign under time inversion: in some cases (the free case, for instance), it does and in other cases (as recalled above), it doesn't.

\section{The quantum time reversal operator: unitary or anti-unitary?}

The quantum definition of a time-reversal operation is

$\psi(\vec{r}, t) \rightarrow \mathbf{T} \tau \psi(\vec{r}, t)$

In this definition, $\mathbf{T}$ is a matrix while $\tau$ acts on $\psi(\vec{r}, t)$ to give rise to

$\psi(\vec{r},-t)$

if the time-reversal operator $T \equiv \mathbf{T} \tau$ is unitary and

$\psi^{*}(\vec{r},-t)$

if it is anti-unitary.

As mentioned in the Introduction, it is commonly agreed that $T$ is anti-unitary. However some discordant voices are raised to oppose this choice. For example, Albert [13] and Callender [14] claim 
that time reversal has to be defined just as the classical time reflection (7). Obviously, this is equivalent to considering $T$ as the (unitary) identity operator ( $T$ is then the identity matrix while $\tau$ corresponds to the unitary choice). Other unitary proposals for $T$ can be found in [15] and [16] when the Dirac equation/field is under study.

Despite these few proposals, the majority of physicists are convinced that only the anti-unitarity of $T$ is a valid option. Let us examine their arguments and see what these arguments become in the light of the unitarity of time reversal.

\subsection{Argument 1: the quantum momentum must change sign, like its classical version}

If we accept this point and if we remember that the momentum has been quantized through

$\vec{p}=-i \bar{h} \vec{\nabla}$

and as neither the Dirac constant $\bar{h}\left(=\frac{h}{2 \pi}\right)$ nor the gradient can be transformed by $T$, we are left with one option only: change the sign of $i$, and this is precisely the role of an anti-unitary operator. Moreover, this ensures the invariance of the fundamental relation of quantum mechanics (in the following equation, we write with bold letters the quantities which change sign under the action of T)

$\left[x_{j}, \mathbf{p}_{\mathbf{k}}\right]=\mathbf{i} \bar{h} \delta_{j k}$

Let's come to the counter-argument. As we have seen in the first Section, the classical momentum does not necessarily change sign. So why should the quantum momentum do so? It is also selfconsistent to ask for the status-quo for the momentum and the invariance for the relation (16) since, as well the left as the right member, do not undergo any change of sign with a unitary temporal inversion.

Close to this point, we find Callender's [14] saying that the Ehrenfest theorem implies

$\frac{d<\vec{r}>}{d t}=\frac{1}{m}<\vec{p}>$

and, consequently, the mean value of $\vec{p}$ has to change sign. Besides the fact that Callender himself would say a few years later [17] that he was not totally convinced by this argument, we could mention that, once again, this point is not valid anymore for other choices of Hamiltonians. For instance, the Pauli Hamiltonian leads to

$\frac{d<\vec{r}>}{d t}=\frac{1}{m}<(\vec{p}-q \vec{A})>$

which has no reason to be invariant under $T$.

Ehrenfest's theorem is therefore not invariant under time inversion, whether $T$ is unitary or anti-unitary (and it is clear that to ask for the invariance of all relations is a challenge, and a useless one at that). Nevertheless, we will find back the results associated with anti-unitary $T$ i.e. the invariance of the Ehrenfest theorem in some cases, by passing to unitarity provided we generalize the inner product. This will be done in section 5 .

On the contrary, the fundamental relation (16) of quantum mechanics is invariant, whether $T$ is anti-unitary or unitary

\subsection{Argument 2: a unitary time reversal operator leads to negative energies}

As mentioned in the Introduction, this is the main argument for rejecting a unitary time reversal operator. This was already stated in [1].
In 2016, Robert formally showed [18] that, given the following three assumptions on the Hamiltonian $H$

$$
\left\{\begin{array}{l}
\langle\psi|H| \psi\rangle \geq 0, \forall \psi \\
H \neq 0 \\
T e^{\frac{i}{h} H t} T^{-1}=e^{-\frac{i}{h} H t}
\end{array}\right.
$$

then $T$ is necessarily anti-unitary.

The second assumption is trivial and the third one comes from

$T e^{\frac{i}{h} H t} T^{-1}=e^{T \frac{i}{h} H t T^{-1}}$

implying

$T i H T^{-1}=-i H$

If $T$ is unitary, then it means that it has to anticommute with $H$

$H T=-T H$

Now, $T$ as a symmetry, must preserve the scalar product. It follows that

$\langle\psi \mid H \psi\rangle=\langle T \psi \mid T H \psi\rangle=-\langle T \psi \mid H T \psi\rangle$

And there we are faced with a state $T|\psi\rangle^{2}$ which is of negative energy since $\langle\psi \mid H \psi\rangle \geq 0$, by assumption. Roberts therefore eliminates the fact that $T$ is unitary since this contradicts his first assumption.

Let's come to the counter-argument. Nothing says that all energies must be positive. As mentioned in the Introduction, there is even more and more evidence that negative energy states have to play a prominent role in cosmology. If this observation holds true in the years to come, the unitarity of the time reversal will have to be considered.

\subsection{Argument 3: the invariance of the Schrodinger equation}

If we follow the tradition of having $T$ anti-unitary, the Schrodinger equation

$\mathbf{i} \bar{h} \frac{\partial \psi}{\partial \mathbf{t}}=-\frac{\bar{h}^{2}}{2 m} \Delta \psi+V \psi$

is indeed invariant while the unitary version seems to lead to a variable one.

To better understand, we must go back to the origins and thus to the set of six papers published by Schrödinger in 1926 [19]. The starting point of Schrödinger was to find an equation, similar to that of the waves, whose solutions would be of the plane wave type

$\psi_{1}=A_{1} e^{-\frac{i}{h}(E t-\vec{p} . \vec{x})}, \psi_{2}=A_{2} e^{-\frac{i}{h}(E t+\vec{p} \cdot \vec{x})}, A_{1}, A_{2} \in \mathbb{C}$

After a first proposal, he highlighted what he put forward as the (real) equation of non-relativistic quantum mechanics

$\left(\Delta-\frac{2 m}{\bar{h}^{2}} V\right)^{2} \psi(\vec{r}, t)+\frac{4 m^{2}}{\bar{h}^{2}} \frac{\partial^{2} \psi(\vec{r}, t)}{\partial t^{2}}=0$

or, by the usual factorization of the wavefunction,

$\left(\left(\Delta-\frac{2 m}{\bar{h}^{2}} V\right)^{2}-\frac{4 m^{2}}{\bar{h}^{2}} E^{2}\right) \phi(\vec{r})=0$

This (fourth-order) equation is obviously invariant under time reflection but also admits solutions with negative energies. It is

\footnotetext{
${ }^{2}$ Note that Weinberg [1] mentioned the state $T^{-1}|\psi\rangle$ instead of $T|\psi\rangle$; this is obviously equivalent since $T^{2}=I$.
} 
particularly easy to convince oneself of this by considering the example of the one-dimensional harmonic oscillator for which

$V=\frac{1}{2} m \omega^{2} x^{2}$

and

$\left(\left(\Delta-\frac{m^{2} \omega^{2}}{\bar{h}^{2}} x^{2}\right)^{2}-\frac{4 m^{2}}{\bar{h}^{2}} E^{2}\right) \phi(\vec{r})=0$

Indeed the usual solutions arise

$\phi(x)=\sqrt[4]{\frac{m \omega}{\pi \bar{h} 2^{2 n}(n !)^{2}}} e^{-\frac{m \omega x^{2}}{2 h}} H_{n}\left(\sqrt{\frac{m \omega}{\bar{h}}} x\right)$

but with positive as well as negative energies

$E= \pm \bar{h} \omega\left(n+\frac{1}{2}\right), n=0,1,2, \ldots$

Given the technical difficulties that a fourth-order equation could cause, Schrödinger then proposed a final version that would simplify the calculations

$\left(\Delta-\frac{2 m}{\bar{h}^{2}} V \pm \frac{2 i m}{\bar{h}} \frac{\partial}{\partial t}\right) \psi(\vec{r}, t)=0$

(see Eq (32) of [20]).

The sign ambiguity in front of the time derivative clearly leaves room for both negative and positive energies and thus for the unitarity of $T$ (see argument 2). This means that the complete Schrödinger equation, with the two possible signs in front of the time derivative, is invariant under unitary time inversion. This equation can take a matrix form

$i \bar{h} \sigma_{3} \frac{\partial \psi(\vec{r}, t)}{\partial t}=-\frac{\bar{h}^{2}}{2 m} \Delta \psi(\vec{r}, t)+V \psi(\vec{r}, t)$

( $\sigma_{3}$ is the usual -diagonal- Pauli matrix) or, equivalently

$i \bar{h} \frac{\partial \psi(\vec{r}, t)}{\partial t}=\left(-\frac{\bar{h}^{2}}{2 m} \Delta+V\right) \sigma_{3} \psi(\vec{r}, t)$

As a unitary time reversal operator has to anticommute with $H$ (see Eq. (21)), it can be realized for instance through

$T=\sigma_{2}$

up to a multiplicative constant of norm equal to 1 . The action of this operator is to reverse the components of the $\psi$, i.e., to reverse the signs of the energies.

Let us also mention that the equation (33) has some common points with the supersymmetric version of quantum mechanics [21] such as two components for the Hamiltonian, one of them leading to negative energies, or the presence of an anticommutation relation (Eq. (21)). However, there are not (fermionic) supercharges here. Instead, we notice, taking the one-dimensional harmonic oscillator, that

$H=\left(-\frac{\bar{h}^{2}}{2 m} \frac{d^{2}}{d x^{2}}+\frac{1}{2} m \omega x^{2}\right) \sigma_{3}=\frac{\bar{h} \omega}{2}\left\{A, A^{\dagger}\right\}$

with

$A=\left(\begin{array}{cc}a & 0 \\ 0 & \alpha a^{\dagger}\end{array}\right), A^{\dagger}=\left(\begin{array}{cc}a^{\dagger} & 0 \\ 0 & \beta a\end{array}\right), \alpha \beta=-1$

being defined in terms of the usual annihilation and creation operators

$a=\sqrt{\frac{\bar{h}}{2 m \omega}}\left(\frac{d}{d x}+\frac{m \omega}{\bar{h}} x\right), a^{\dagger}=\sqrt{\frac{\bar{h}}{2 m \omega}}\left(-\frac{d}{d x}+\frac{m \omega}{\bar{h}} x\right)$
The relation of the Heisenberg algebra

$$
\left[A, A^{\dagger}\right]=I
$$

obviously holds true.

We immediately see that the operators (36) are not adjoint to each other, with respect to the usual scalar product.

To see more clearly what is behind this, let us go to the following Section.

\section{A unifled approach from both perspectives}

So far, we have developed arguments related to the unitarity of the time reversal operator to highlight negative energies.

We claim now that we can see similar results i.e. the emergence of negative energy states, by rotating spatial coordinates.

To enlighten this statement, we use the method of complex coordinate rotation first introduced with a real exponential (related to the dilatation group $[22,23]$ ) and then extended to a complex exponential (for a review, see [24]) acting on the spatial coordinates:

$x \rightarrow x(\theta)=e^{i \theta} x$

This change of variables has been introduced in a different context than the one considered here i.e. the study of quasi-bound resonance states having a complex spectrum.

Nevertheless, we use this new coordinate system here to define generalized annihilation and creation operators through

$a(\theta) \equiv \sqrt{\frac{\bar{h}}{2 m \omega}}\left(e^{-i \theta} \frac{d}{d x}+e^{i \theta} \frac{m \omega}{\bar{h}} x\right)=\sqrt{\frac{\bar{h}}{2 m \omega}}\left(\frac{i}{\bar{h}} p(\theta)+\frac{m \omega}{\bar{h}} x(\theta)\right)$

$$
\begin{aligned}
a^{\dagger}(\theta) & =\sqrt{\frac{\bar{h}}{2 m \omega}}\left(-e^{-i \theta} \frac{d}{d x}+e^{i \theta} \frac{m \omega}{\bar{h}} x\right) \\
& =\sqrt{\frac{\bar{h}}{2 m \omega}}\left(-\frac{i}{\bar{h}} p(\theta)+\frac{m \omega}{\bar{h}} x(\theta)\right)
\end{aligned}
$$

It is straightforward to convince ourselves that

$\left[a(\theta), a^{\dagger}(\theta)\right]=1$

as well as

$\frac{\bar{h} \omega}{2}\left\{a(\theta), a^{\dagger}(\theta)\right\}=H(\theta)=-e^{-2 i \theta} \frac{\bar{h}^{2}}{2 m} \frac{d^{2}}{d x^{2}}+\frac{1}{2} e^{2 i \theta} m \omega^{2} x^{2}$

It is then clear that the positive-energy Hamiltonian corresponds to $\theta=0$ (or $x(\theta)=x$ ) while the negative-energy one is related to $\theta=\frac{\pi}{2}$ (or $\left.x(\theta)=i x\right)$.

Solving

$H(\theta) \phi=E \phi$

we are led to

$E=-\bar{h} \omega \epsilon\left(n+\frac{1}{2}\right), n=0,1,2, .$.

and the usual eigenstates (29). Here $\epsilon$ is defined according to

$\epsilon=-1$ if $\theta=0, \epsilon=1$ if $\theta=\frac{\pi}{2}$

We thus recover the results (29)-(30) of the real (fourth-order) equation.

As the complex rotation method has no impact on the eigenvectors for the oscillator potential, we can introduce a new inner product 
Table 1

The different features of anti-unitary $T$ as well as unitary $T$.

\begin{tabular}{ll}
\hline Anti-unitary $T$ & Unitary $T$ \\
\hline$i \rightarrow-i$ & $i \rightarrow i$ \\
$\vec{p} \rightarrow-\vec{p}$ & $\vec{p} \rightarrow \vec{p}$ \\
$E \geq 0$ & $E \geq 0(\theta=0) \leftrightarrow E \leq 0\left(\theta=\frac{\pi}{2}\right)$ \\
\hline
\end{tabular}

whose only change is related to the transformation of the spatial coordinate

$\langle\psi \mid \phi\rangle_{\theta} \equiv e^{-i \theta} \int \psi(x)^{*} \phi(x) d x$

When positive energies are concerned $(\theta=0)$, we recover the usual Hermitian inner product while it becomes skew-Hermitian for negative energies $\left(\theta=\frac{\pi}{2}\right.$ ). Skew-Hermiticity is actually what characterizes the negative-energies annihilation and creation operators since

$a\left(\frac{\pi}{2}\right)=\sqrt{\frac{1}{2 m \omega h}}(p+i m \omega x), a^{\dagger}\left(\frac{\pi}{2}\right)=\sqrt{\frac{1}{2 m \omega h}}(-p+i m \omega x)$

and

$p\left(\frac{\pi}{2}\right)=-\frac{d}{d x}, x\left(\frac{\pi}{2}\right)=i x$

Note that the presence of the phase factor in this new inner product does not change the probabilistic interpretation of quantum mechanics since transition probabilities are given par the product of the inner product by its complex conjugate.

We can also come back to the Ehrenfest theorem by mentioning that the mean values of the position and momentum operators now lead to

$\langle x(\theta)\rangle_{\theta}=e^{-i \theta} e^{i \theta} \int \psi^{*} x \psi d x=\langle x\rangle$

$\langle p(\theta)\rangle_{\theta}=e^{-i \theta} e^{-i \theta} \int \psi^{*} p \psi d x=-\langle p\rangle$ if $\theta=\frac{\pi}{2}$

As a consequence, this new inner product allows us to find, in the unitary context, the sign change of the mean value of $p$, typical of the anti-unitary context.

Notice also that the idea of a new scalar product is not new. It has already been evoked in [25] (where we can find a mixed scalar product, the usual scalar product for the angular part of the Schrödinger equation and the scalar product without complex conjugation for the radial part) and [26] (in which a scalar product based on a time inversion only is introduced).

\section{Conclusion}

We have highlighted some arguments that allow us to affirm that the choice of anti-unitarity for time inversion is not as obvious as it seemed at first sight..

Let us resume the different characteristics in a summary table (Table 1 ).

In both cases, the fundamental relations and equations of nonrelativistic quantum mechanics remain invariant. Of course, this is a first step and it is necessary to confirm it at the level of relativistic quantum mechanics and quantum field theory.

At least, both versions, unitary and anti-unitary, are coherent, from a theoretical point of view if we limit ourselves to non-relativistic quantum mechanical considerations.

To the reader worried about experimental confirmations, we can only refer to the fact that negative energies, and thus the unitary temporal reversal operator, have already proven themselves at the cosmological scale [10]. It should also be noted that the field of investigation of negative energies and masses is recent. Further confirmation is clearly needed in the future. Experiments such as those conducted in [27] will be crucial in this sense. Our goal, in this article, was simply to bring a first stone to the theoretical building of this new aspect of physics by limiting ourselves to the foundations i.e. non-relativistic quantum mechanics.

\section{CRediT authorship contribution statement}

\section{J.-P. Petit: Conceptualization.}

\section{Declaration of competing interest}

The authors declare that they have no known competing financial interests or personal relationships that could have appeared to influence the work reported in this paper.

\section{Acknowledgements}

Warm thanks are due to the members of the Support au Modèle Cosmologique Janus for their financial support.

\section{References}

[1] Steven Weinberg. The Quantum Theory of Fields, Cambridge University Press, 1995, p. 76.

[2] Adam G. Riess, et al, Astron. J. 116 (1998) 1009.

[3] H. Bondi, Rev. Mod. Phys. 29 (3) (1957) 423.

[4] J. Belletête, M.B. Paranjape, Int. J. Mod. Phys. D 22 (2013) 1341017.

[5] S. Mbarek, M.B. Paranjape, Phys. Rev. D 90 (2014) 101502(R).

[6] R.T. Hammond, Eur. J. Phys. 36 (2015) 025005.

[7] V.V. Dvoeglazov, Z. Naturforsch. A 71 (2016) 345.

[8] J.-P. Petit, G. d'Agostini, Mod. Phys. Lett. A 29 (2014) 1450182

[9] J.-P. Petit, G. d'Agostini, Astrophys. Space Sci. 363 (2018) 139.

[10] J.-P. Petit, G. d'Agostini, N. Debergh, Janus cosmological model, ffhal-03285671, 2021.

[11] E. Wigner, Group Theory and Its Applications to the Quantum Mechanics of Atomic Spectra, Academic Press, 1931.

[12] U. Uhlhorn, Ark. Fys. 23 (1963) 307.

[13] D.Z. Albert, Time and Chance, Harvard University Press, 2000.

[14] C. Callender, Is time "handed" in a quantum world?. Proc. Aristot. Soc. 247 (2000).

[15] N. Debergh, J.-P. Petit, G. d'Agostini, J. Phys, Commun. 2 (2018) 115012.

[16] Frédéric Henry-Couannier, Glob. J. Sci. Front. Res. 12 (2012) 39.

[17] C. Callender, Quantum Mechanics: Keeping it Real? 2020, preprint July 23rd.

[18] Bryan W. Roberts, Philos. Sci. 84 (2016) 315.

[19] E. Schrödinger, Ann. Phys. 384 (1926) 361.

[20] E. Schrödinger, Phys. Rev. 28 (1926) 1049.

[21] E. Witten, Nucl. Phys. B 188 (1981) 513.

[22] E. Balslev, J.M. Combes, Commun. Math. Phys. 22 (1971) 296.

[23] B. Simon, Commun. Math. Phys. 27 (1972) 1.

[24] Y.K. Ho, Phys. Rep. 99 (1983) 1.

[25] N. Moiseyev, C. Corcoran, Phys. Rev. A 20 (1979) 614.

[26] F. Weinhold, Symmetry 13 (2021) 808.

[27] M.A. Khamehchi, et alls, Phys. Rev. Lett. 118 (2017) 155301. 